\title{
Hyponatremia and Congestive Heart Failure: A Marker of Increased Mortality and a Target for Therapy
}

\author{
Adam Romanovsky, ${ }^{1}$ Sean Bagshaw, ${ }^{2}$ and Mitchell H. Rosner ${ }^{3}$ \\ ${ }^{1}$ Divsions of Nephrology and Critical Care, University of Alberta, Edmonton, AB, Canada T6G 2G3 \\ ${ }^{2}$ Division of Critical Care, University of Alberta, Edmonton, AB, Canada T6G $2 G 3$ \\ ${ }^{3}$ Divsion of Nephrology, University of Virginia Health System, Charlottesville, Box 800133, VA 22911, USA
}

Correspondence should be addressed to Mitchell H. Rosner, mhr9r@virginia.edu

Received 2 August 2010; Accepted 23 February 2011

Academic Editor: Anjay Rastogi

Copyright (c) 2011 Adam Romanovsky et al. This is an open access article distributed under the Creative Commons Attribution License, which permits unrestricted use, distribution, and reproduction in any medium, provided the original work is properly cited.

Heart failure is one of the most common chronic medical conditions in the developed world. It is characterized by neurohormonal activation of multiple systems that can lead to clinical deterioration and significant morbidity and mortality. In this regard, hyponatremia is due to inappropriate and continued vasopressin activity despite hypoosmolality and volume overload. Hyponatremia is also due to diuretic use in an attempt to manage volume overload. When hyponatremia occurs, it is a marker of heart failure severity and identifies patients with increased mortality. The recent introduction of specific vasopressin-receptor antagonists offers a targeted pharmacological approach to these pathophysiological derangements. Thus far, clinical trials with vasopressin-receptor antagonists have demonstrated an increase in free-water excretion, improvement in serum sodium, modest improvements in dyspnea but no improvement in mortality. Continued clinical trials with these agents are needed to determine their specific role in the treatment of both chronic and decompensated heart failure.

\section{Introduction}

Heart failure (HF) is an increasingly common condition, with recent American data revealing a 1 in 5 lifetime risk for both sexes, and over 5 million currently affected patients [1]. In addition to being common, morbidity and mortality attributable to HF continue to rise with over 1.1 million hospital discharges and 1 in 8 death certificates mentioning heart failure in 2006 [1]. HF has an annual mortality of $20 \%$ per year after diagnosis, and its estimated economic burden in the United Sates in 2009 was $\$ 37.2$ billion [1].

Although HF manifests primarily with cardiopulmonary symptoms, hyponatremia is very common in this patient population. In fact, hyponatremia (variably defined as serum sodium $<1342-136 \mathrm{mmol} / \mathrm{L}$ ) is present in over $20 \%$ of patients admitted to hospital with HF $[2,3]$. Not only is it a common occurrence, but it has repeatedly been shown to be a marker of increased mortality in the HF population $[3,4]$.
As will be discussed in this paper, both the maladaptive neurohormonal and renal changes as well as diuretic treatment of HF contribute to the development of hyponatremia [2-4]. In particular, the posterior pituitary hormone vasopressin leads to renal water retention and hyponatremia. As such, the recently developed vasopressin antagonists present an attractive target for the management of hyponatremia in HF [5].

\section{Physiology of Water Handling}

To appreciate the pathophysiology of hyponatremia in HF, it is important to understand the basic physiology of renal salt and water handling. With the exception of psychogenic polydipsia and low dietary solute intake, essentially all cases of true hyponatremia represent a failure to excrete maximally dilute urine. In the presence of normal renal function, this 
failure is most often related to the action of vasopressin (AVP).

AVP is a hormone synthesized in the supraoptic (SON) and paraventricular nuclei of the hypothalamus and is released from the posterior pituitary [6]. Its effects are multiple and related to the affected receptor (Table 1). Binding to the $V_{\text {la }}$ receptor leads to vascular smooth muscle contraction [7] while $V_{2}$ receptor activation in the renal medulla leads to free water reabsorption by the collecting duct. Binding to $V_{2}$ receptors, located on the basolateral membrane of the cortical collecting duct cells, leads to increased aquaporin 2 (aqp-2) mRNA levels and translocation of aqp-2 to the apical membranes [6]. This increases tubular water permeability and allows water to move from the tubule to the medullary interstitium (down a concentration gradient), resulting in net reabsorption of free water. This movement of water is passive and relies upon a hypertonic renal medulla, the generation of which is partly dependant on the activity of the NKCC (sodium-potassium-2 chloride) channels in the ascending loop of henle [8]. Absence of AVP activity (such as in diabetes insipidus) leads to loss of high volume dilute urine.

AVP release is mediated by both osmotic as well as cardiac output and intravascular volume stimuli. Osmoreceptors present in the SON are exceedingly sensitive to changes in serum osmolality demonstrating alterations in AVP release in response to a $1 \%$ fluctuation in serum osmolality [9]. This sensitivity serves to keep serum osmolality tightly controlled with a threshold for AVP release of approximately $280 \mathrm{mOsm} / \mathrm{Kg}$ [9]. The nonosmotic stimuli for AVP release consist of reductions in cardiac output, intravascular blood volume, or blood pressure [9]. These stimuli, mediated through high (aortic arch and carotid sinus) and low (left atrial) pressure baroreceptors [10], enhance the secretion of AVP for any given osmotic stimulus $[9,10]$. In effect, AVP will be released at a lower plasma osmolality when decreased intravascular volume, cardiac output or blood pressure are detected.

Recently, AVP has been shown to be a potent and independent regulator of the thiazide-sensitive $\mathrm{Na}(+)-\mathrm{Cl}(-)$ cotransporter (NCC) [11, 12]. This action is mediated through the $\mathrm{V}_{2}$-receptor and its clinical effects are, as yet, not clear but implicate AVP in the handling of sodium as well as water.

\section{Pathophysiology of Hyponatremia in Heart Failure}

In the normal physiologic state, alterations in serum osmolality serve as the primary control for AVP release. However, in conditions that lead to nonosmotic stimulation of AVP release, these stimuli may take precedence resulting in the acceptance of a lower serum osmolality [9]. Such is the case in HF where a decrease in cardiac output leads to a continued release AVP despite a reduction in osmolality, thus leading to hyponatremia. Multiple studies have demonstrated increased levels of AVP in HF leading accompanied by inadequate inhibition when exposed to a decrease in serum osmolality
$[13,14]$. Indeed, data in the Studies of Left Ventricular Dysfunction (SOLVD) show a progressive incremental increase in AVP levels with worsening HF symptoms [15]. It has also been shown that the density of AVP positive neurons in the SON is increased by as much as $30 \%$ in patients with HF [16].

A number of other neurohormonal abnormalities contribute to abnormalities in renal sodium $(\mathrm{Na})$ and water handling. Arterial underfilling (from decreased cardiac output) detected by baroreceptors in the aortic arch, carotid sinus and afferent renal arterioles leads to activation of the sympathetic nervous system (SNS) and the renin-angiotensinaldosterone system (RAAS) [2]. While the activation of these systems acts to preserve systemic perfusion pressure in patients with HF, it is a long-term maladaptive response that leads to avid $\mathrm{Na}$ and water retention in multiple nephron segments. This overload of $\mathrm{Na}$ and water may worsen cardiac function thus perpetuating the cycle of $\mathrm{Na}$ and water retention. In addition, angiotensin II is a potent inhibitor of thirst, which may lead to increased free water intake and exacerbation of hyponatremia [17].

Reductions in glomerular filtration rate are common in patients with $\mathrm{HF}$ and can eventually lead to a reduced capacity for water and $\mathrm{Na}$ excretion. It has been shown that the filtered load of $\mathrm{Na}$ decreases in parallel with declining GFR in patients receiving diuretics [18]. While salt intake in these patients exacerbates volume overload and HF, they are also at risk of worsening hyponatremia with increased free water intake.

Although a mainstay in the therapy of HF, diuretics can also cause hyponatremia. These drugs increase $\mathrm{Na}$ and water excretion thereby alleviating congestive symptoms and theoretically helping to optimize cardiac contractility. It is interesting to note that despite their widespread use, diuretics have not been shown to improve survival in HF patients [19]. Diuretics are prescribed in $85-100 \%$ of symptomatic and 16-35\% asymptomatic patients with reduced left ventricular function [20-22]. Loop diuretics are the most commonly utilized diuretics and exert their salt wasting effects by inhibiting the NKCC channel in the thick ascending loop of Henle. Other diuretics used frequently in HF include thiazide diuretics and spironolactone. Thiazides inhibit the $\mathrm{Na}-\mathrm{Cl}$ cotransporter in the distal convoluted tubule while spironolactone prevents activation of the mineralocorticoid receptor on the principal cells of the cortical collecting duct. Of these 3 classes, loop diuretics offer the most potent increase in $\mathrm{Na}$ and water excretion and thus are important agents in the treatment of states of volume overload.

In the general population, diuretic-induced hyponatremia is very common, with thiazides accounting for the $63 \%$ of the cases of severe hyponatremia, loop diuretics for $6 \%$, and spironolactone for $1 \%$ [23]. The incidence of hyponatremia with thiazide diuretics may be as high as $11 \%$ in the elderly [24]. Several features may contribute to hyponatremia: (1) stimulation of AVP release secondary to diuretic-induced volume contraction, (2) decrease in GFR from intravascular volume contraction, (3) inhibition of urinary dilution capacity due to interference with $\mathrm{Na}$ absorption in the distal segments, and (4) hypokalemia induced intracellular shift of $\mathrm{Na}$ [24]. The thiazide effect in the distal 
TABLE 1: The actions of vasopressin and its receptors.

\begin{tabular}{lll}
\hline Receptor & Location & Action \\
\hline & Vascular smooth muscle & Vasoconstriction \\
$\mathrm{V}_{\mathrm{la}}$ & Platelets & Myocardial hypertrophy \\
& Myometrium & Aggregation \\
\hline $\mathrm{V}_{\mathrm{lb}}$ & Anterior pituitary & Uterine contraction \\
\hline & Renal collecting tubule & Adrenocorticotropin hormone release \\
$\mathrm{V}_{2}$ & & Induction of aquaporin-2 \\
& Vascular endothelium & Free water absorption \\
& & Release of von Willebrand factor \\
\hline
\end{tabular}

nephron accounts for its association with hyponatremia. Conversely, loop diuretics may be spared from causing hyponatremia by their effect on the NKCC co-transporter, which helps maintain the hypertonic medullary interstitium. A reduction in the tonicity in this area decreases the gradient for free water movement out of the tubules via aqp-2 channels and may therefore lessen the risk of hyponatremia as compared to thiazides.

Owing to their disruption of the medullary concentration gradient, loop diuretics may actually lead to an increase in $\mathrm{Na}$ in hyponatremic patients [25]. If, however, there is incomplete amelioration of the concentration gradient, administration of loop diuretics can still cause hyponatremia. This likely relates to further stimulation of the RAAS due to increased distal Na delivery thereby increasing angiotensin II, a well-known stimulant of AVP secretion [26].

\section{Epidemiology of Hyponatremia in Heart Failure}

Given the number of neurohormonal changes in patients with HF, it is not surprising that hyponatremia is very common in this population. With $\mathrm{Na}$ and water retention and decreased GFR from activation of the RAAS and SNS in the context of increased AVP levels, continued intake of hypotonic fluids may lead to hyponatremia. Of all patients admitted to hospital with a diagnosis of HF, $18-27 \%$ will have hyponatremia $(\mathrm{Na}<135 \mathrm{mmol} / \mathrm{L})$ on admission [27, 28].

Not only is hyponatremia common, it is also a strong marker of increased morbidity and mortality in HF patients. Lee and Packer [29] analyzed 30 clinical, hemodynamic, and biochemical variables and their association with survival in 203 consecutive patients with severe HF. The most powerful predictor of cardiovascular mortality was pretreatment serum $\mathrm{Na}$, with hyponatremic patients having a substantially shorter median survival than patients with a normal serum $\mathrm{Na}$ (164 versus 373 days, $P=.006$ ). Similarly, in the Outcomes of a Prospective Trial of Intravenous Milrinone for Exacerbations of Chronic Heart Failure (OPTIME-CHF) study, both in hospital and 60-day mortality rates were highest for patients with the lowest admission serum $\mathrm{Na}$ [27]. In the Organized Program to Initiate Lifesaving Treatment in Hospitalized Patients with Heart Failure (OPTIMIZEHF) registry, patients with hyponatremia had significantly higher in-hospital and follow-up mortality rates and longer hospital stays [30]. In this study, for each $3 \mathrm{mmol} / \mathrm{L}$ decrease in serum $\mathrm{Na}$ below $140 \mathrm{mmol} / \mathrm{L}$ at admission, the risk of in-hospital mortality and follow-up mortality increased by $19.5 \%$ and $10 \%$, respectively. More recently, the importance of persistent hyponatremia in HF patients was described in a cohort of patients enrolled in the Evaluation Study of Congestive Heart Failure and Pulmonary Artery Catheterization Effectivenes (ESCAPE) [31]. Hyponatremia in this study was associated with higher 6-month mortality after covariate adjustment (hazard ratio(HR), for each $3 \mathrm{mmol} / \mathrm{L}$ decrease in serum Na, 1.23 ; $95 \%$ confidence interval (CI), 1.05-1.43; $P=.01$ ). After controlling for baseline variables and clinical response, patients with persistent hyponatremia had an increased risk of all-cause mortality (31\% versus $16 \%$; HR, 1.82; $P=.04)$, HF rehospitalization ( $62 \%$ versus $43 \%$; HR, 1.52; $P=.03$ ), and death or rehospitalization (73\% versus $50 \%$; HR, $1.54 ; P=.01)$ compared with normonatremic patients.

It is unclear whether this increased mortality directly related to hyponatremia or if the sodium abnormality is a marker of a more severe underlying illness. It is likely that it reflects a greater activation of the RAAS and SNS with higher levels of AVP leading to increased mortality. It has in fact been shown that patients with hyponatremia have higher circulating levels of catecholamines, renin, angiotensin, aldosterone, and AVP [32]. It is also feasible that the presence of hyponatremia limits options in terms of diuretic management and could potentially alter HF therapy leading to differences in mortality. Moreover, hyponatremia associated with diuretic use may be accompanied by multiple other metabolic abnormalities such as hypokalemia and hypomagnesemia that could increase mortality. In addition, severe hyponatremia and its correction can respectively lead to cerebral edema and the osmotic demyelination syndrome, both of which are associated with high morbidity and mortality.

\section{Management of Hyponatremia in Heart Failure}

Management of hyponatremia in heart failure demands a multifaceted approach including optimization of cardiac function (including prevention of volume overload and neurohormonal blockade), preservation of renal function, 
and maintenance of appropriate fluid intake. In addition, the relatively newly developed vasopressin antagonists potentially offer an attractive therapeutic strategy for dealing with hyponatremia in HF. Of utmost importance in any patient with hyponatremia is to ensure adequate monitoring of serum Na levels, as rapid changes in either direction can have dire consequences.

5.1. Optimization of Cardiac Function. Perhaps a simplistic view of managing hyponatremia in HF is to ensure adequate cardiac output. Ideally, this would reduce the stimulation of baroreceptors and lessen the activation of the SNS and RAAS resulting in less renal avidity for $\mathrm{Na}$ and water and lower levels of AVP.

While detailed management of HF is beyond the scope of this paper, inhibition of the SNS and RAAS with $\beta$ blockers (BB) and angiotensin converting enzyme inhibitors (ACEi) or angiotensin receptor blockers (ARB) remain the cornerstone of chronic HF management [33]. These agents break the maladaptive cycle of neurohormonal activation and via multiple mechanisms lead to improved preservation of cardiac function [33]. They also lead to a reduction in left ventricular afterload facilitating an improvement in cardiac output. In addition, blockade of the aldosterone action with spironolactone or eplerenone decreases hospitalizations and mortality in patients with New York Heart Association (NYHA) class III and IV HF $[34,35]$. In patients with acute exacerbations of $\mathrm{HF}$, afterload reduction with ACEi and nitrates and utilization of positive inotropic agents may be used to improve cardiac output [36] thus increasing Na levels in hyponatremic patients.

As previously discussed, diuretics remain a mainstay of HF treatment and have complex effects on serum $\mathrm{Na}$ levels. Increasing $\mathrm{Na}$ and water loss can ease congestive symptoms and, especially in combination with afterload reduction and increased inotropy, can improve cardiac output in the volume-overloaded patient [36]. This is usually accomplished with loop diuretics, or a combination of loop and thiazide diuretics, and can lead to increased $\mathrm{Na}$ levels in the hyponatremic patient. It is important to note, however, that excessive diuresis leads to hypovolemia, activation of the SNS and RAAS, and decreased renal function. This effect can worsen cardiac function and lead to impaired renal $\mathrm{Na}$ and water handling resulting in hyponatremia from increased AVP release. In addition, a single bolus of furosemide has been associated with an increase in plasma renin activity, norepinephrine, and AVP leading to increased left ventricular filling pressure and decreased stroke volume [37]. This response to furosemide is potentially detrimental to the HF patient.

Diuretic use has been associated with increased mortality in both chronic and acute exacerbations of HF [38, 39]. It is difficult, however, to delineate a cause and effect relationship, and despite a lack of evidence for their effectiveness, diuretics will likely remain an important component of $\mathrm{HF}$ management for the foreseeable future. Due to the complex physiology of $\mathrm{HF}$, the effect of loop diuretics on serum $\mathrm{Na}$ can be difficult to accurately predict thus making frequent monitoring of serum $\mathrm{Na}$ very important.
In addition, nonpotassium sparing diuretics may lead to significant hypokalemia, hypomagnesemia, and decreased renal function. It is therefore prudent to ensure adequate monitoring of these parameters when using these agents.

5.2. Preservation of Renal Function. Patients with impaired renal function have a decreased capacity for $\mathrm{Na}$ and water excretion thus placing them at an increased risk of developing hyponatremia. Efforts to maintain normal renal function including blood pressure control, limiting use of nephrotoxic medications and contrast dye, and avoiding excessive diuresis may help to limit the risk of hyponatremia. In patients whose renal function is sufficiently poor to maintain appropriate $\mathrm{Na}$ and water balance, renal replacement therapy (hemodialysis or peritoneal dialysis) can remove excessive $\mathrm{Na}$ and water and maintain normal sodium levels.

5.3. Maintenance of Appropriate Fluid Intake. With high levels of circulating AVP, HF patients will have a limited capacity to excrete excess dietary free water. It thus follows that HF patients with hyponatremia should limit dietary water intake. The degree of limitation necessary will be patient specific and dictated by the degree of neurohormonal activation in each patient. Again, frequent monitoring will help ensure an appropriate rise in serum $\mathrm{Na}$ in response to the intervention.

5.4. Vasopressin Antagonists. Given the primary role of AVP in free water retention and the development of hyponatremia, antagonism of AVP action would seem like a rational therapeutic option in hyponatremic HF patients. As vasopressin also leads to vasoconstriction and cardiomyocyte hypertrophy [7], blocking its actions may have further beneficial effects in HF.

A number of such drugs have been developed and target either $\mathrm{V}_{2}$ receptors selectively or a combination of $\mathrm{V}_{2}$ or $\mathrm{V}_{\text {la }}$ receptors. These agents lead to a selective loss in renal free water losses termed aquaresis. To date, no studies have shown a reduction in mortality with use of the vasopressor antagonists in HF.

Preclinical studies in animals and humans showed that administration of a $\mathrm{V}_{2}$ receptor antagonist leads to an increase in free water excretion with little increased $\mathrm{Na}$ loss and no compensatory activation of the RAAS [40-42]. These positive results have led to a number of clinical trials of these agents in HF patients.

Georghiade et al. compared tolvaptan to placebo in 254 NYHA class III or IV outpatients who continued to receive standard HF therapy [43]. Patients received either 1 of 3 oral doses of tolvaptan $(30,45$, or $60 \mathrm{mg} /$ day) or placebo for a total of 25 days. Although all patients treated with tolvaptan had an increase in serum $\mathrm{Na}$, the $28 \%$ who had baseline had hyponatremia the greatest rise. $80 \%$ of tolvaptan-treated patients with hyponatremia had normalization of serum $\mathrm{Na}$ on day 1 compared to $40 \%$ of those receiving placebo. These patients also had significant reductions in body weight with an improvement in HF symptoms. A similar study in 319 patients randomized patients to 1 of 3 tolvaptan doses $(30,60$, or $90 \mathrm{mg})$ or placebo, in addition to standard HF 
treatment for 60 days [28]. Patients in the tolvaptan group had small increases in serum $\mathrm{Na}$; the greatest rise in $\mathrm{Na}$ was seen in the $21.3 \%$ of patients with baseline hyponatremia. Tolvaptan-treated patients also had a significant decrease in body weight at 24 hours (median $2.05 \mathrm{Kg}$ in the highest dose group) with no changes in heart rate, blood pressure, renal function, or development of hypokalemia.

A larger study of 4133 patients assessed both- short and long-term outcomes in patients admitted with acutely decompensated HF $[44,45]$. Patients were randomized to receive Tolvaptan $30 \mathrm{mg} /$ day or placebo in addition to standard HF care. Short-term outcomes at 7 days revealed no difference in the primary outcome of global clinical status. Similar to the previous studies, however, the tolvaptan group did have a significant decrease in body weight and dyspnea. Of note, treatment with tolvaptan also led to significant increases in thirst, polyuria, and hypernatremia $(1.4 \%$ versus $0 \%)$. The long-term follow-up trial over a mean of 9.9 months, found no difference in all-cause mortality, cardiovascular death, or HF hospitalizations between the groups. Improvements in dyspnea and serum $\mathrm{Na}$ were maintained throughout followup with similar side effects to those seen in the short-term trial. A subgroup analysis of the $8 \%$ of the patients with baseline hyponatremia found serum $\mathrm{Na}$ to be increased by $5.5 \mathrm{mmol} / \mathrm{L}$ and $1.8 \mathrm{mmol} / \mathrm{L}$ in the tolvaptan and placebo groups, respectively.

Similar short-term outcomes of increased urine output and serum sodium were found in patients with stable HF receiving lixivaptan, another $\mathrm{V}_{2}$-receptor antagonist [46]. Ascending single doses of the drug were used and produced a dose-dependent increase in urine output but no long-term outcomes with this agent have been published.

Antagonism of the $\mathrm{V}_{1 \mathrm{a}}$ receptors in addition to the $\mathrm{V}_{2}$ receptors has the added theoretical benefit of decreasing afterload by inhibiting AVP-mediated smooth muscle contraction. Conivaptan is one such agent with FDA approval for treatment of hypervolemic hyponatremia. In a randomized, double-blind, placebo-controlled, multicenter study, 84 patients with hyponatremia were randomized to receive 1 of 2 doses of conivaptan $(20 \mathrm{mg}$ bolus followed by 96 hour infusion of either 40 or $80 \mathrm{mg} /$ day) or placebo, in addition to standard HF treatments [47]. Both doses of conivaptan were associated with significant increases in serum $\mathrm{Na}$. $\mathrm{Na}$ levels increased by $6 \mathrm{mmol} / \mathrm{L}$ or were normalized in $69 \%$ of the $40 \mathrm{mg} /$ day dose and $88.5 \%$ of the $80 \mathrm{mg} /$ day dose by day 4 , whereas only $20.7 \%$ of the placebo group achieved this goal.

Several studies of conivaptan in HF patients have yielded similar results to those with $\mathrm{V}_{2}$-receptor antagonists [4850]. Recipients of conivaptan had increases in urine output with decrease in left- and right-sided filling pressures and had minimal side effects. When compared with furosemide alone, a combination of furosemide and conivaptan produced a dose-dependent increase in urine output [50]. In addition, combination with higher doses of conivaptan $(80$ or $120 \mathrm{mg} /$ day) led to small but significant increases in serum $\mathrm{Na}$.

The precise role of vasopressin antagonists in the management of hyponatremic HF patients remains unclear. While no effects on mortality have been seen in clinical trials, there are clear improvements in symptoms in the majority of studies. To date, there have been no studies directly comparing the effects of $\mathrm{V}_{2}$ and combined $\mathrm{V}_{2} / \mathrm{V}_{1 \mathrm{a}}$-recpetor antagonists which remains an interesting clinical question. It is important to note that in all of the aforementioned studies, vasopressin antagonists have been used in conjunction with usual HF treatment (including diuretics), and have not been studied as a replacement for loop diuretics.

\section{Conclusion}

Hyponatremia in HF is a frequent occurrence related to the activation of a multitude of neurohormonal pathways including the SNS, RAAS, and particularly the increased release of AVP. In addition to being common, hyponatremia is associated with increased mortality in the HF population. The treatment has traditionally consisted of RAAS and SNS blockade in combination with loop and thiazide diuretics and dietary water restriction. While this approach can be effective, diuretics have several detrimental metabolic side effects and may potentially worsen hyponatremia and cardiac function. Vasopressin antagonism represents a logical goal in the management of hyponatremia in the HF population. These agents have been shown to increase serum $\mathrm{Na}$ and free water clearance and improve HF symptoms, but have not yet been found to reduce long-term mortality. More trials are necessary to define an exact role for the vasopressin antagonists in HF patients.

\section{References}

[1] W. Rosamond, K. Flegal, K. Furie et al., "Heart disease and stroke statistics-2008 update: a report from the American heart association statistics committee and stroke statistics subcommittee," Circulation, vol. 117, no. 4, pp. e25-e46, 2008.

[2] D. A. Sica et al., "Sodium and water retention in heart failure and diuretic therapy: basic mechanisms," Cleveland Clinic Journal of Medicine, vol. 73, supplement 2, pp. S30-S33, 2006.

[3] A. Goldberg, H. Hammerman, S. Petcherski et al., "Hyponatremia and long-term mortality in survivors of acute STelevation myocardial infarction," Archives of Internal Medicine, vol. 166, no. 7, pp. 781-786, 2006.

[4] L. De Luca, L. Klein, J. E. Udelson et al., "Hyponatremia in patients with heart failure," The American Journal of Cardiology, vol. 96, no. 12, pp. 19L-23L, 2005.

[5] C. R. Lee, M. L. Watkins, J. H. Patterson et al., "Vasopressin: a new target for the treatment of heart failure," American Heart Journal, vol. 146, no. 1, pp. 9-18, 2003.

[6] S. E. Ishikawa and R. W. Schrier, "Pathophysiological roles of arginine vasopressin and aquaporin-2 in impaired water excretion," Clinical Endocrinology, vol. 58, no. 1, pp. 1-17, 2003.

[7] S. R. Goldsmith, "Vasopressin as vasopressor," American Journal of Medicine, vol. 82, no. 6, pp. 1213-1219, 1987.

[8] S. Nielsen, T. H. Kwon, B. M. Christensen, D. Promeneur, J. Frøkiær, and D. Marples, "Physiology and pathophysiology of renal aquaporins," Journal of the American Society of Nephrology, vol. 10, no. 3, pp. 647-663, 1999.

[9] F. L. Dunn, T. J. Brennan, A. E. Nelson, and G. L. Robertson, "The role of blood osmolality and volume in regulating 
vasopressin secretion in the rat," The Journal of Clinical Investigation, vol. 52, no. 12, pp. 3212-3219, 1973.

[10] P. H. Baylis, "Posterior pituitary function in health and disease," Clinics in Endocrinology and Metabolism, vol. 12, no. 3, pp. 747-770, 1983.

[11] N. B. Pedersen, M. V. Hofmeister, L. L. Rosenbaek, J. Nielsen, and R. A. Fenton, "Vasopressin induces phosphorylation of the thiazide-sensitive sodium chloride cotransporter in the distal convoluted tubule," Kidney International, vol. 78, no. 2, pp. 160-169, 2010.

[12] K. Mutig, T. Saritas, S. Uchida et al., "Short-term stimulation of the thiazide-sensitive $\mathrm{Na}+\mathrm{C} 1-$ cotransporter by vasopressin involves phosphorylation and membrane translocation," American Journal of Physiology, vol. 298, no. 3, pp. F502-F509, 2010.

[13] B. F. Uretsky, J. G. Verbalis, and T. Generalovich, "Plasma vasopressin response to osmotic and hemodynamic stimuli in heart failure," American Journal of Physiology, vol. 17, no. 3, pp. H396-H402, 1985.

[14] S. R. Goldsmith, G. S. Francis, and A. W. Cowley, "Arginine vasopressin and the renal response to water loading in congestive heart failure," American Journal of Cardiology, vol. 58, no. 3, pp. 295-299, 1986.

[15] G. S. Francis, C. Benedict, D. E. Johnstone et al., "Comparison of neuroendocrine activation in patients with left ventricular dysfunction with and without congestive heart failure. A substudy of the studies of left ventricular dysfunction (SOLVD)," Circulation, vol. 82, no. 5, pp. 1724-1729, 1990.

[16] E. V. Sivukhina, I. U. E. Morozov, A. A. Dolzhikov, G. F. Jirikowski, and V. Grinevich, "Comparison of vasopressin and oxytocin expressions in the hypothalamo-neurohypophysial system of patients with chronic heart failure," Hormone and Metabolic Research, vol. 42, no. 1, pp. 56-60, 2010.

[17] A. Kazory, "Hyponatremia in heart failure: revisiting pathophysiology and therapeutic strategies," Clinical Cardiology, vol. 33, no. 6, pp. 322-329, 2010.

[18] D. A. Sica and T. W. B. Gehr, "Diuretic combinations in refractory oedema states: pharmacokinetic-pharmacodynamic relationships," Clinical Pharmacokinetics, vol. 30, no. 3, pp. 229 249, 1996.

[19] B. K. Krämer, F. Schweda, and G. A. J. Riegger, "Diuretic treatment and diuretic resistance in heart failure," American Journal of Medicine, vol. 106, no. 1, pp. 90-96, 1999.

[20] K. Swedberg, U. Idanpaan-Heikkila, and J. Remes, "Effects of enalapril on mortality in severe congestive heart failure. Results of the cooperative north scandinavian enalapril survival study (CONSENSUS)," The New England Journal of Medicine, vol. 316, no. 23, pp. 1429-1435, 1987.

[21] S. Yusuf, "Effect of enalapril on survival in patients with reduced left ventricular ejection fractions and congestive heart failure," The New England Journal of Medicine, vol. 325, no. 5, pp. 293-302, 1991.

[22] S. Yusuf, J. M. Nicklas, G. Timmis et al., "Effect of enalapril on mortality and the development of heart failure in asymptomatic patients with reduced left ventricular ejection fractions," The New England Journal of Medicine, vol. 327, no. 10, pp. 685-691, 1992.

[23] M. Sonnenblick, Y. Friedlander, and A. J. Rosin, "Diureticinduced severe hyponatremia review and analysis of 129 reported patients," Chest, vol. 103, no. 2, pp. 601-606, 1993.

[24] P. A. Sarafidis, P. I. Georgianos, and A. N. Lasaridis, "Diuretics in clinical practice. Part II: electrolyte and acid-base disorders complicating diuretic therapy," Expert Opinion on Drug Safety, vol. 9, no. 2, pp. 259-273, 2010.
[25] A. Kazory, "Hyponatremia in heart failure: revisiting pathophysiology and therapeutic strategies," Clinical Cardiology, vol. 33, no. 6, pp. 322-329, 2010.

[26] G. S. Francis, R. M. Siegel, and S. R. Goldsmith, "Acute vasoconstrictor response to intravenous furosemide in patients with chronic congestive heart failure. Activation of the neurohumoral axis," Annals of Internal Medicine, vol. 103, no. 1, pp. 1-6, 1985.

[27] L. Klein, C. M. O'Connor, J. D. Leimberger et al., "Lower serum sodium is associated with increased short-term mortality in hospitalized patients with worsening heart failure: results from the outcomes of a prospective trial of intravenous milrinone for exacerbations of chronic heart failure (OPTIMECHF) study," Circulation, vol. 111, no. 19, pp. 2454-2460, 2005.

[28] M. Gheorghiade, W. A. Gattis, C. M. O'Connor et al., "Effects of tolvaptan, a vasopressin antagonist, in patients hospitalized with worsening heart failure: a randomized controlled trial," Journal of the American Medical Association, vol. 291, no. 16, pp. 1963-1971, 2004.

[29] W. H. Lee and M. Packer, "Prognostic importance of serum sodium concentration and its modification by convertingenzyme inhibition in patients with severe chronic heart failure," Circulation, vol. 73, no. 2, pp. 257-267, 1986.

[30] M. Gheorghiade, W. T. Abraham, N. M. Albert et al., "Relationship between admission serum sodium concentration and clinical outcomes in patients hospitalized for heart failure: an analysis from the OPTIMIZE-HF registry," European Heart Journal, vol. 28, no. 8, pp. 980-988, 2007.

[31] M. Gheorghiade, J. S. Rossi, W. Cotts et al., "Characterization and prognostic value of persistent hyponatremia in patients with severe heart failure in the ESCAPE trial," Archives of Internal Medicine, vol. 167, no. 18, pp. 1998-2005, 2007.

[32] L. S. Lilly, V. J. Dzau, and G. H. Williams, "Hyponatremia in congestive heart failure: implications for neurohumoral activation and responses to orthostasis," Journal of Clinical Endocrinology and Metabolism, vol. 59, no. 5, pp. 924-930, 1984.

[33] G. V. Ramani, P. A. Uber, and M. R. Mehra, "Chronic heart failure: contemporary diagnosis and management," Mayo Clinic Proceedings, vol. 85, no. 2, pp. 180-195, 2010.

[34] B. Pitt, F. Zannad, W. J. Remme et al., "The effect of spironolactone on morbidity and mortality in patients with severe heart failure," The New England Journal of Medicine, vol. 341, no. 10, pp. 709-717, 1999.

[35] B. Pitt, W. Remme, F. Zannad et al., "Epleronone post-acute myocardial infarction heart failure efficacy and survival study investigators. Epleronone, a selective aldosterone blocker, in patients with left ventricular dysfunction after myocardial infarction," The New England Journal of Medicine, vol. 351, pp. 543-551, 2004.

[36] D. D. Shin, F. Brandimarte, L. De Luca et al., "Review of current and investigational pharmacologic agents for acute heart failure syndromes," American Journal of Cardiology, vol. 99, no. 2, pp. S4-S23, 2007.

[37] G. S. Francis, R. M. Siegel, S. R. Goldsmith et al., "Acute vasoconstrictor response to intravenous furosemide in patients with chronic congestive heart failure. Activation of the neurohumoral axis," Annals of Internal Medicine, vol. 103, no. 1, pp. 1-6, 1985.

[38] A. Ahmed, A. Husain, T. E. Love et al., "Heart failure, chronic diuretic use, and increase in mortality and hospitalization: an observational study using propensity score methods," European Heart Journal, vol. 27, no. 12, pp. 1431-1439, 2006. 
[39] V. Hasselblad, W. G. Stougha, M. R. Shahb et al., "Relation between diuretic dose and outcome in a heart failure population: results of the ESCAPE trial," Journal of Cardiac Failure, vol. 9, no. 10, pp. 1064-1069, 2005.

[40] A. Ohnishi, Y. Orita, N. Takagi et al., "Aquaretic effect of a potent, orally active, nonpeptide $\mathrm{V}$ antagonist in men," Journal of Pharmacology and Experimental Therapeutics, vol. 272, no. 2, pp. 546-551, 1995.

[41] L. M. Burrell, P. A. Phillips, J. Risvanis, R. K. Chan, K. L. Aldred, and C. I. Johnston, "Long-term effects of nonpeptide vasopressin $V_{2}$ antagonist OPC-31260 in heart failure in the rat," American Journal of Physiology, vol. 275, no. 1, pp. H176H182, 1998.

[42] T. Hirano, Y. Yamamura, S. Nakamura, T. Onogawa, and T. Mori, "Effects of the $V_{2}$-receptor antagonist OPC-41061 and the loop diuretic furosemide alone and in combination in rats," Journal of Pharmacology and Experimental Therapeutics, vol. 292, no. 1, pp. 288-294, 2000.

[43] M. Georghiade et al., "Tolvaptan Investigators. Vasopressin $V_{2}$-recepto blockadw tih tovaptan in patients with chronic heart failure: results from a double-blind randomized control trial," Circulation, vol. 107, pp. 2690-2696, 2003.

[44] M. Gheorghiade, M. A. Konstam, J. C. Burnett Jr. et al., "Short-term clinical effects of tolvaptan, an oral vasopressin antagonist, in patients hospitalized for heart failure: the EVEREST clinical status trials," Journal of the American Medical Association, vol. 297, no. 12, pp. 1332-1343, 2007.

[45] M. A. Konstam, M. Gheorghiade, J. C. Burnett Jr. et al., "Effects of oral tolvaptan in patients hospitalized for worsening heart failure: the EVEREST outcome trial," Journal of the American Medical Association, vol. 297, no. 12, pp. 1319-1331, 2007.

[46] W. T. Abraham, A. A. Shamshirsaz, K. McFann, R. M. Oren, and R. W. Schrier, "Aquaretic effect of lixivaptan, an oral, nonpeptide, selective V2 receptor vasopressin antagonist, in New York heart association functional class II and III chronic heart failure patients," Journal of the American College of Cardiology, vol. 47, no. 8, pp. 1615-1621, 2006.

[47] D. Zeltser, S. Rosansky, H. Van Rensburg, J. G. Verbalis, and N. Smith, "Assessment of the efficacy and safety of intravenous conivaptan in euvolemic and hypervolemic hyponatremia," American Journal of Nephrology, vol. 27, no. 5, pp. 447-457, 2007.

[48] J. E. Udelson, W. B. Smith, G. H. Hendrix et al., "Acute hemodynamic effects of conivaptan, a dual $\mathrm{V}$ and $\mathrm{V}$ vasopressin receptor antagonist, in patients with advanced heart failure," Circulation, vol. 104, no. 20, pp. 2417-2423, 2001.

[49] S. R. Goldsmith, U. Elkayam, W. H. Haught, A. Barve, and W. $\mathrm{He}$, "Efficacy and safety of the vasopressin V1A/V2-receptor antagonist conivaptan in acute decompensated heart failure: a dose-ranging pilot Study," Journal of Cardiac Failure, vol. 14, no. 8, pp. 641-647, 2008.

[50] S. R. Goldmsith, U. Elkayam, B. McNutt et al., "Combined effects of conivaptan and furosemide on urine output in patients with acute decompensated heart failure," Critical Care Medicine, vol. 36, p. A61, 2008. 


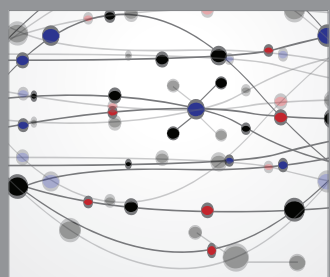

The Scientific World Journal
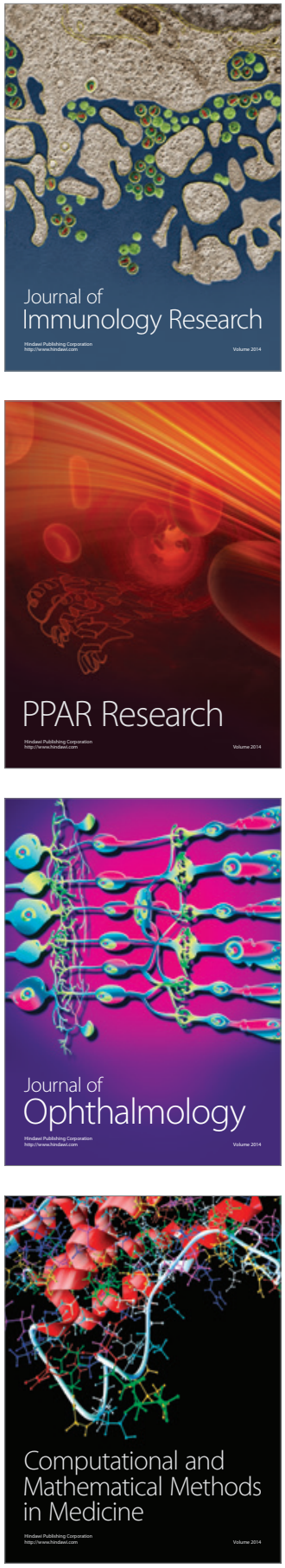

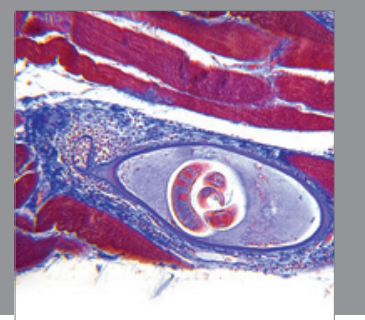

Gastroenterology

Research and Practice
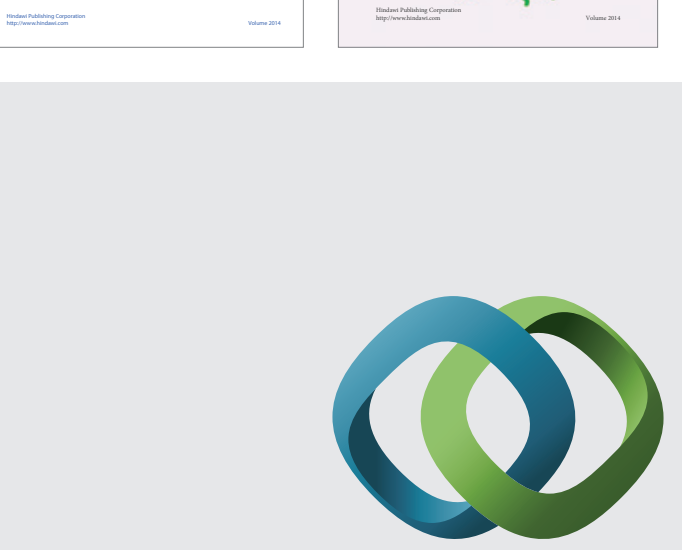

\section{Hindawi}

Submit your manuscripts at

http://www.hindawi.com
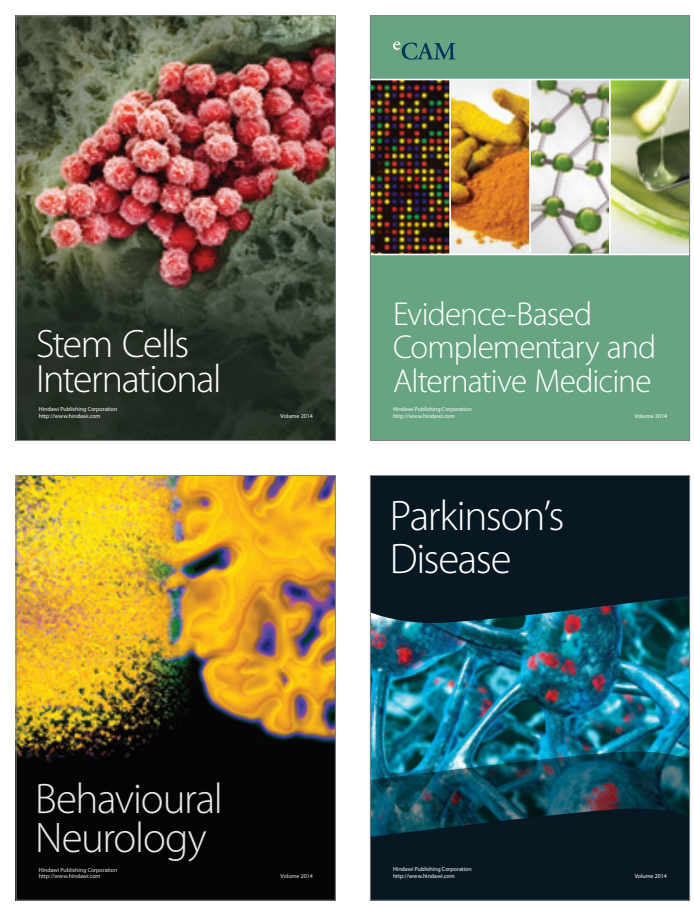

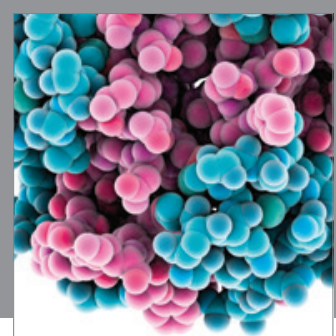

Journal of
Diabetes Research

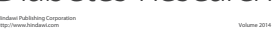

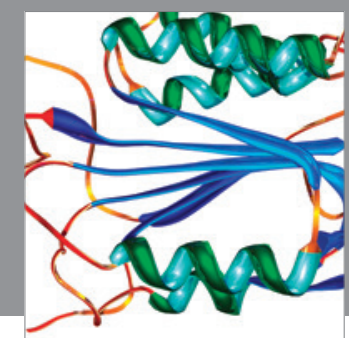

Disease Markers
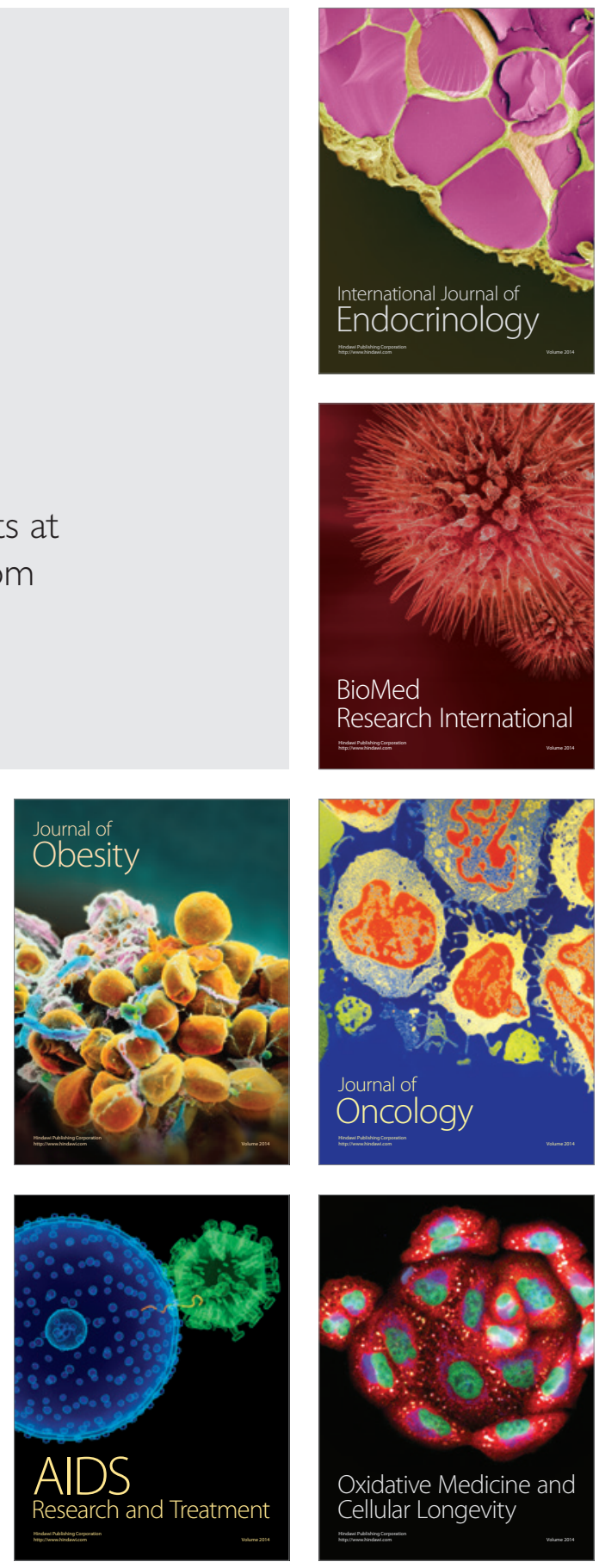